\title{
研究論文
}

\section{Application of the Response Matrix Method to Criticality Calculations of One-dimensional Reactors}

\author{
応答行列法による 1 次元原子炬臨界計算 \\ By Akinao SHIMIZU*, Kazuo MONTA* and Toshiki MIYAMOTO*
}

\begin{abstract}
The response matrix method has successfully been applied to problems to solve the multigroup diffusion equation for one-dimensional multiregion reactors. The method consists of the calculation of response matrices of spatial regions, which is reduced to a boundary value problem for a bare homogeneous region and can be performed analytically, and of the criticality calculation by means of response matrices, which can be performed by simple algebra involving matrices even for reactors consisting of many spatial regions, since the order of matrices involved is equal to the number of energy groups independently of the number of spatial regions. By a numerical calculation it has been confirmed that the method gives us the accurate solution and that the execution time of the machine is appreciably less than that by the finite difference method for few-group calculations. Criticality calculations by means of an electronic analog computer were also performed. It was shown that the analog calculation gives us the multiplication factor of two-region reactors with an error less than $0.1 \%$ by simple procedures.
\end{abstract}

\section{INTRODUCTION}

In the preceding paper ${ }^{(1)}$ we developed the response matrix method for estimating the criticality of the reactor from the viewpoint of "a black box".

In the present paper we shall apply the response matrix method to a mathematical problem - to solve the multigroup diffusion equation for one-dimensional reactors.

Let us consider a one-dimensional reactor consisting of several spatial regions. It is assumed that the composition in any spatial region is homogeneous although it changes from region to region. The multigroup diffusion equation for this reactor is a set of differential equations of the second order and can in principle be solved analytically. However, for more than a few regions or energy groups, such a solution seems to be too cumbersome because of the large order determinants involved.
Recently numerical methods for solving the multigroup diffusion equation by the use of high-speed digital computers have been developed $^{(2)(3)}$ and play an important part in the design of reactors.

In numerical calculations we usually reduce the differential equation to a linear algebraic equation which can be solved by the computing machine. According to the finite difference method $^{(2)}$ which has been used in most numerical solutions, the differential equation is reduced to a difference equation for flux.

According to the response matrix method we reduce the differential equation to another linear algebraic equation which is of the form

$\boldsymbol{J}^{-}(m-1)=\boldsymbol{R}^{+}(m) \cdot \boldsymbol{J}^{+}(m-1)+\boldsymbol{T}^{-}(m) \cdot \boldsymbol{J}^{-}(m)$ $\boldsymbol{J}^{+}(m)=\boldsymbol{T}^{+}(m) \cdot \boldsymbol{J}^{+}(m-1)+\boldsymbol{R}^{-}(m) \cdot \boldsymbol{J}^{-}(m)$,

* 清水彰直, 門田一雄, 宮本俊樹。NAIG Nucl. Res. Lab. (日本原子力事業(株)NAIG棇合研究所) 
where $\boldsymbol{J}^{+}(m)$ and $\boldsymbol{J}^{-}(m)$ are column vectors the orders of which are equal to the number of energy groups. The $n$-th component of the vector $\boldsymbol{J}^{+}(m), j_{n}^{+}(m)$, represents the current of the energy group $n$ going to the right direction at the interface between the region $m$ and $(m+1)$, and $j_{\bar{n}}(m)$ represents the current going toward the left.

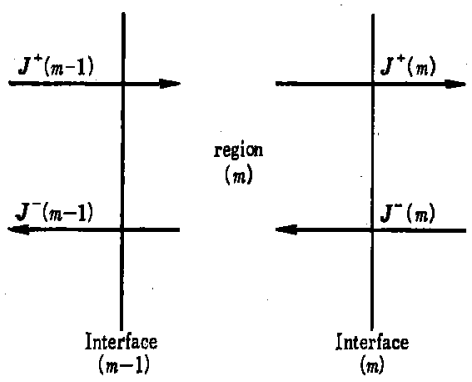

Fig. I Response of one-dimensional region

These currents are assumed to be expressed by a linear combination of flux of the group $n, \phi_{n}$ and its derivative $\phi_{n}^{\prime}$ as

$$
j_{n}^{ \pm}(m)=\frac{1}{4}\left\{\phi_{n}(m) \mp 2 D_{n} \cdot \phi_{n}^{\prime}(m)\right\},
$$

where $D_{n}$ represents the diffusion coefficient for the group $n$.

$\boldsymbol{R}^{ \pm}(m)$ and $\boldsymbol{T}^{ \pm}(m)$ are square matrices, called the response matrices, and their orders are equal to the number of energy groups. It is an important feature of the present method that the response matrices $\boldsymbol{R}^{ \pm}(m)$ and $\boldsymbol{T}^{ \pm}(m)$ are dependent only on the composition and the geometry of the region $m$. The physical basis of this feature was established in the preceding paper ${ }^{(1)}$. This is reasonable mathematically also, since the solution of a set of differential equations of the second order with $G$ unknown functions is uniquely determined by $2 G$ boundary conditions.

Eq. (1) has the following feature compared with the difference equation:

(1) The calculation of the response matrix may be more cumbersome than that of the coefficients in the difference equation. It is, however, reduced to a boundaryvalue problem for a homogeneous bare region and can be carried out analytically unless the number of energy groups is too large.
(2) The unknown quantities in Eq. (1) are only the currents at interfaces between spatial regions. Hence in most cases the number of unknown variables is much reduced compared with that in the difference equation.

(3) The present reduction is rigorous, whereas the reduction in the difference equation is only approximately valid.

(4) Eq.(1) can be solved as easily as or more easily than the difference equation unless the number of energy grours is too large.

(5) Eq. (1) is also applicable to a region, such as a control plate or a cavity, to which diffusion approximation can not be applicable, provided that the response matrix of such a region is determined by a more refined theory.

(6) The response matrix has a clear physical meaning and can in principle be determined directly by measurements.

\section{Calculation of Response Matrix}

We shall calculate the response matrices of an infinite homogeneous slab of thickness $a$ in the $G$-group diffusion approximation. Assuming that only a steady incident current of the group $m$ with a unit intensity is introduced into the slab through the surface at $x=0$ (Fig. 2), we denote the stationary flux of the group $n$ produced by this incident current inside the slab by $\phi_{n, m}(x)$, the current of the group $n$ going to the right direction by $j_{n, m}^{+}(x)$, and the current to the left by $j_{\bar{n}, m}(x)$. According to the $G$-group diffusion approximation, the neutron flux satisfies equations

$$
\left.\begin{array}{c}
-D_{n} \frac{d^{2}}{d x^{2}} \phi_{n, m}+\left(\Sigma_{e n}+\Sigma_{s n}\right) \cdot \phi_{n, m} \\
=\Sigma_{s n^{-1}} \cdot \phi_{n-1, m}+S_{n, m} \\
S_{n, m}=\chi_{n} \cdot \sum_{n^{\prime}} \nu \Sigma_{f^{\prime}} \cdot \phi_{n^{\prime}, m} \\
n=1,2, \cdots \cdots, G,
\end{array}\right\}
$$

where $D_{n}, \Sigma_{a n}, \Sigma_{s n}, \Sigma_{f n}, \nu$ and $\chi_{n}$ are the group constants and represent the diffusion coefficient for neutrons in the group $n$, the absorption cross-section, the slowing down 
cross-section, the fission cross-section, the number of neutrons liberated per fission, and the normalized fission spectrum, respectively.

The netutron current is expressed in terms of the flux in the form

$$
j_{n, m}^{ \pm}(x)=\frac{1}{4}\left\{\phi_{n, m}(x) \mp d_{n} \cdot \phi_{n, m}^{\prime}(x)\right\},
$$

where $d_{n}=2 D_{n}$ and $\phi_{n, m}^{\prime} \equiv(d / d x) \phi_{n, m}$.

We assume that Eq. (4) holds at every point in the slab, even at the outer boundary. Although this assumption seems crude, it is essential in the calculation of the response matrix with the diffusion approximation because we should always expect that the outer surface of the slab under consideration will be an interface between two slabs by the contact of another slab. In our method the boundary condition at the interface is that the current going to the right direction and that to the left are respectively continuous. This condition is equivalent to the condition usually used that flux and net current are respectively continuous, as far as Eq. (4) is satisfied at the interface.

One obtains $G$ material bucklings $\left(B_{r}^{2} ; \mu=\right.$ $1,2, \cdots, G)$ by the condition that a set of linear homogeneous equations

$$
\begin{gathered}
\left(D_{n} \cdot B_{\mu}^{2}+\sum_{a n}+\sum_{s n}\right) \cdot \alpha_{n}^{(\mu)}-\sum_{n n-1} \cdot \alpha_{n-1}^{(\mu)} \\
-\chi_{n} \cdot \sum_{n^{\prime}} \nu \Sigma_{f n^{\prime}} \cdot \alpha_{n^{\prime}}^{(\mu)}=0
\end{gathered}
$$

has non-trivial solutions. Corresponding to each material buckling $B_{\mu}^{2}$, one gets a set of the coefficients $\left(\alpha_{n}^{(\mu)} ; n=1,2, \cdots \cdots, G\right)$ so called the coupling constants and two characteristic functions $\boldsymbol{\varphi}_{\mu}^{+}(x)$ and $\boldsymbol{\phi}_{\mu}^{-}(x)$ which satisfy an equation

$$
\frac{d^{2}}{d x^{2}} \varphi_{\mu}+B_{\mu}^{2} \cdot \varphi_{\mu}=0
$$

Then a solution of Eq. ( 3 ) is expressed by a linear combination of those $2 G$ functions as

$$
\begin{gathered}
\phi_{n, m}=\sum_{\mu=1}^{o} \alpha_{n}^{(\mu)} \cdot\left\{C_{\mu}^{+}(m) \cdot \phi_{\mu}^{+}+C_{\mu}^{-}(m) \cdot \phi_{\mu}^{-}\right\} \\
n=1,2, \cdots \cdots, G,
\end{gathered}
$$

where $C_{\boldsymbol{F}}^{ \pm}(m)$ are undetermined coefficients. Index $m$ in $C_{\mu}^{ \pm}(m)$ indicates that they are the coefficients for the flux produced by the incident current of the group $m$.

Those $2 G$ coefficients are determined by $2 G$ boundary conditions for $\phi_{n, m}$. They are:

$$
j_{n, m}^{-}(a)=\frac{1}{4}\left\{\phi_{n, m}(a)+d_{n} \cdot \phi_{n, m}^{\prime}(a)\right\}=0 .
$$

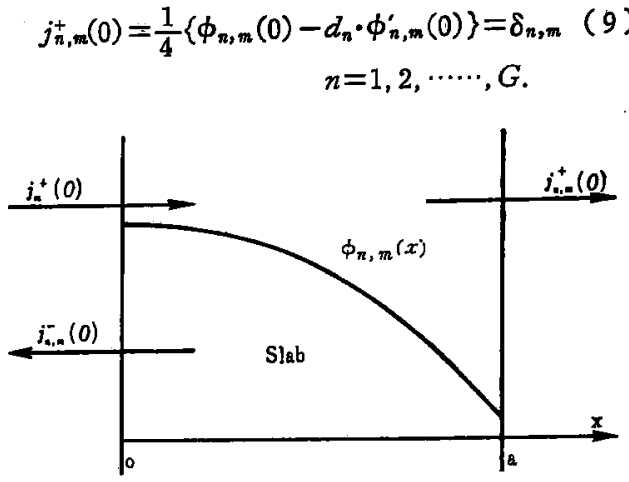

Fig. 2 Current and flux in a slab

Then the desired response matrices viewed from the surface $x=0$ are obtainable by equations

$$
\begin{aligned}
& R_{n, m}^{+}=\frac{1}{4}\left\{\phi_{n, m}(0)+d_{n} \cdot \phi_{n, m}^{\prime}(0)\right\}, \\
& T_{n, m}^{+}=\frac{1}{4}\left\{\phi_{n, m}(a)-d_{n} \cdot \phi_{n, m}^{\prime}(a)\right\} .
\end{aligned}
$$

The response matrices viewed from the other surface of the slab $\boldsymbol{R}^{-}$and $\boldsymbol{T}^{-}$are respectively equal to $\boldsymbol{R}^{+}$and $\boldsymbol{T}^{+}$for a homogeneous slab.

The determination of the coefficients $C_{m}^{ \pm}(m)$ can be performed by calculating determinants of the order $2 G$.

We shall next show that the order of the determinants involved can be reduced to $G$ by a slight modification.

We first calculate an approximate flux $\tilde{\phi}_{n, m}(x)$ by replacing the boundary condition (8) with an approximate one

$$
\phi_{n, m}(a+\delta)=0 \quad n=1,2, \cdots \cdots, G .
$$

where $\delta$ represents the extrapolated boundary which is assumed common to all energy groups. With the above condition one can easily eliminate $G$ coefficients between $2 G$ coefficients in Eq. (7) and will get an expression

$$
\bar{\phi}_{n, m}(x)=\sum_{\mu=1}^{a} C_{\mu}(m) \cdot \alpha_{n}^{(\mu)} \cdot \phi_{\mu},
$$

where $\phi_{\mu}$ represents the characteristic function satisfying both Eq. ( 6 ) and the boundary. condition (12).

Let us define approximate response matrices. $\tilde{\boldsymbol{R}}^{+}$and $\tilde{\boldsymbol{T}}^{+}$by equations

$$
\begin{aligned}
& \tilde{\boldsymbol{R}}_{n, m}^{+}=\frac{1}{4}\left\{\tilde{\phi}_{n, m}(0)+d_{n} \cdot \tilde{\phi}_{n, m}^{\prime}(0)\right\}, \\
& \tilde{\boldsymbol{T}}_{n, m}^{+}=\frac{1}{4}\left\{\tilde{\phi}_{n, m}(a)-d_{n} \cdot \tilde{\phi}_{n, m}^{\prime}(a)\right\}
\end{aligned}
$$


and an error current matrix $W^{+} b_{\text {, }}$ an equation

$$
W_{n, m}^{+}=\frac{1}{4}\left\{\tilde{\phi}_{n, m}(a)+d_{n} \cdot \bar{\phi}_{n, m}^{\prime}(a)\right\} .
$$

The calculation of the above matrices is reduced to that of a determinants of the order $G$.

In the same way we define the approximate response matrices $\tilde{\boldsymbol{R}}^{-}$and $\tilde{\boldsymbol{T}}^{-}$and the error current matrix $\boldsymbol{W}^{-}$viewed from the other surface of the slab. Then the following equations hold between those approximate matrices and the rigorous one:

$$
\begin{aligned}
& \tilde{\boldsymbol{R}}^{+}=\boldsymbol{R}^{+}+\boldsymbol{T}^{-} \cdot \boldsymbol{W}^{+} \\
& \tilde{T}^{+}=\boldsymbol{T}^{+}+\boldsymbol{R}^{-} \cdot \boldsymbol{W}^{+} \\
& \tilde{\boldsymbol{R}}^{-}=\boldsymbol{R}^{-}+\boldsymbol{T}^{+} \cdot \boldsymbol{W}^{-} \\
& \tilde{T}^{-}=T^{-}+\boldsymbol{R}^{+} \cdot \boldsymbol{W}^{-}
\end{aligned}
$$

Solving the above four equations with respect to $\boldsymbol{R}^{ \pm}$and $\boldsymbol{T}^{ \pm}$, one finally gets equations

$$
\left.\begin{array}{l}
\boldsymbol{R}^{+}=\left(\tilde{\boldsymbol{R}}^{+}-\tilde{\boldsymbol{T}}^{-} \boldsymbol{W}^{+}\right) \cdot\left(\boldsymbol{E}-\boldsymbol{W}^{-} \cdot \boldsymbol{W}^{+}\right)^{-1} \\
\boldsymbol{T}^{+}=\left(\tilde{\boldsymbol{T}}^{+}-\tilde{\boldsymbol{R}}^{-} \cdot \boldsymbol{W}^{+}\right) \cdot\left(\boldsymbol{E}-\boldsymbol{W}^{-} \cdot \boldsymbol{W}^{+}\right)^{-1} \\
\boldsymbol{R}^{-}=\left(\tilde{\boldsymbol{R}}^{-}-\tilde{\boldsymbol{T}}^{+} \boldsymbol{W}^{-}\right) \cdot\left(\boldsymbol{E}-\boldsymbol{W}^{+} \cdot \boldsymbol{W}^{-}\right)^{-1} \\
\boldsymbol{T}^{-}=\left(\tilde{\boldsymbol{T}}^{-}-\tilde{\boldsymbol{R}}^{+} \cdot \boldsymbol{W}^{-}\right) \cdot\left(\boldsymbol{E}-\boldsymbol{W}^{+} \cdot \boldsymbol{W}^{-}\right)^{-1}
\end{array}\right\}
$$

Eq. (14) is valid not only to the slab.but also to a one-dimensional region of other geometry. Especially for a homogeneous slab, Eqs. (14) are reduced to

$$
\begin{aligned}
& R^{ \pm}=[\tilde{\boldsymbol{R}}-\tilde{\boldsymbol{T}} \cdot \boldsymbol{W}] \cdot\left(\boldsymbol{E}-W^{2}\right)^{-1} \\
& \boldsymbol{T}^{ \pm}=[\tilde{\boldsymbol{T}}-\tilde{\boldsymbol{R}} \cdot W] \cdot\left(E-W^{2}\right)^{-1},
\end{aligned}
$$

where $\tilde{\boldsymbol{R}}=\tilde{\boldsymbol{R}}^{+}=\tilde{\boldsymbol{R}}^{-}, \quad \tilde{\boldsymbol{T}}=\tilde{\boldsymbol{T}}^{+}=\tilde{\boldsymbol{T}}^{-}$and $\boldsymbol{W}=\boldsymbol{W}^{+}$ $=W^{-}$.

The response matrices of an infinite annulus and a spherical shell are obtainable in the same way as those of a slab.

\section{Criticality Calculation}

We shall next calculate the multiplication factor and the neutron current at every interface between spatial regions by solving Eq. (1). The reactor under consideration is assumed to consist of $\left(M+M^{i}\right)$ regions. We divide the reactor into two sub-assemblies, the right sub-assembly consisting of $M$ regions and the left one consisitng of $M^{\prime}$ regions, by a suitable interface and give a number to each region and interface as shown in Fig. 3.

Eq. (1) can be solved by comparatively

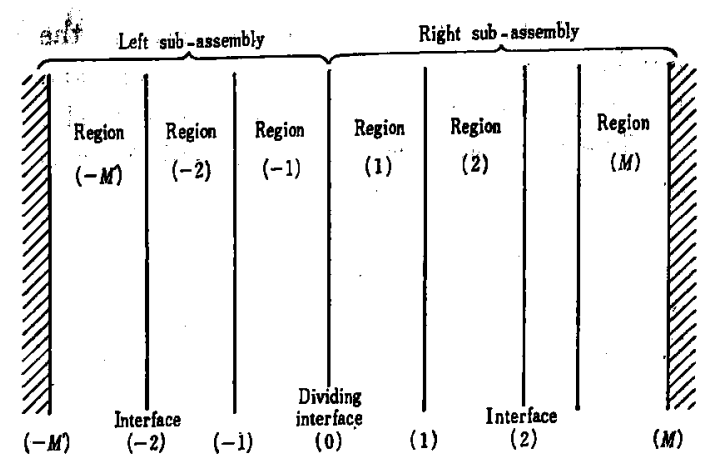

Fig. 3 Regions in one-dimensional reactor

simple algebra involving matrix of the order $G$ as follows:

According to the combination rule derived in the preceding paper ${ }^{(1)}$, the reflection matrix of a combined system of regions $(m),(m+1)$, ... and $(M)$ in the right sub-assembly viewed from the interface $(m-1)$, which we denote by $\boldsymbol{R}_{m+1}^{+}$, is proved to satisfy a recurrence formula

$$
\begin{aligned}
\boldsymbol{R}_{m+1}^{+}= & \boldsymbol{R}^{+}(m)+\boldsymbol{T}^{-}(m) \cdot\left\{\boldsymbol{E}-\boldsymbol{R}_{m}^{+} \cdot \boldsymbol{R}^{-}(\boldsymbol{m})\right\}^{-1} \\
& \cdot \boldsymbol{R}_{m}^{+} \cdot \boldsymbol{T}^{+}(m),
\end{aligned}
$$

where $\boldsymbol{E}$ is the unit matrix and $\boldsymbol{A}^{-1}$ represents the inverse of a matrix $\boldsymbol{A}$. By the formula (16) with the initial condition

$$
\boldsymbol{R}_{\text {I }-1}^{+}=\boldsymbol{R}^{+}(M)
$$

one obtains the reflection matrix of the right sub-assmbly $\boldsymbol{R}_{0}^{+}$viewed from the dividing interface. Similary the reflection matrix of the left sub-assembly viewed from the dividing interface $\boldsymbol{R}_{0}^{-}$is obtainable. Then the multiplication factor and the current at the dividing interface is determined by solving the equation derived in the preceding paper ${ }^{(1)}$

$$
\begin{aligned}
& \left(\boldsymbol{R}_{0}^{-} \cdot \boldsymbol{R}_{0}^{+}-\lambda \boldsymbol{E}\right) \cdot \boldsymbol{J}^{+}(o)=0 \\
& \boldsymbol{J}^{-}(o)=\boldsymbol{R}_{0}^{+} \cdot \boldsymbol{J}^{+}(o) .
\end{aligned}
$$

Once the current at the dividing interface is given, one will get the current at every interface by equations

$$
\begin{aligned}
\boldsymbol{J}^{-}(m)= & \left\{\boldsymbol{T}^{+}(m)\right\}^{-1} \cdot\left\{\boldsymbol{J}^{-}(m-1)\right. \\
& \left.-\boldsymbol{R}^{+}(m) \cdot \boldsymbol{J}^{+}(m-1)\right\} \\
\boldsymbol{J}^{+}(m)= & \boldsymbol{T}^{+}(m) \cdot \boldsymbol{J}^{+}(m-1) \\
& +\boldsymbol{R}^{-}(m) \cdot \boldsymbol{J}^{-}(m) .
\end{aligned}
$$

The above equations are derived by a slight modification of Eq. (1).

If the neutron flux in the region $(m)$ is required, it is given by an equation 


$$
\begin{aligned}
\Psi(x, m)= & \boldsymbol{F}^{+}(\boldsymbol{x}, m) \cdot \boldsymbol{J}^{+}(m-1) \\
& +\boldsymbol{F}^{-}(x, m) \cdot \boldsymbol{J}^{-}(m),
\end{aligned}
$$

where $\boldsymbol{F}^{ \pm}(\boldsymbol{x}, m)$ represent the flux matrices of the region $(m)$ which can be calculated by a similar method as that for the calculation of the response matrices.

The multiplication factor and the flux thus determined are in accord with those by a diffusion code based on the finite difference method for the just critical reactor. There exists, however, some difference between those quantities by both methods for a noncritical reactor. This is due to the difference in the definition of the generation of neutrons between two methods. Since the eigenvalues of a reactor calculated by both methods are not the dynamic criticality factor defined rigorously by the persistent distribution in a non-critical reactor but static $^{(4)}$ criticality factors introduced by a strategem to reduce a time-dependent problem for a non-critical reactor to a stationary problem from the viewpoint of a generation of neutrons, an eigenvalue and a corresponding distribution by a definition of the generation of neutrons are not always in accord with those by another definition.

The rigorous treatment of a non-critical reactor by the response matrix method is also possible and was shown in the preceding paper $^{(1)}$. It can be performed by replacing the response matrix in the above-mentioned process of calculations with Laplace transform of the time-dependent response matrices.

\section{Numerical Illustrations}

A two group diffusion code for slab reactors consisting of many spatial regions by the response matrix method MERMAIDIA has been programmed for USSC-90*.

A typical execution time of this code is $4 \mathrm{sec}$ for a calculation of response matrices of a region and $(2 \cdot M)$ sec for a calculation of the multiplication factor and the mean flux in every region, where $M$ represents the number of spatial regions in a reactor.

In order to confirm the response matrix method three kinds of check calculations have been performed.

\section{Check calculation 1}

We first calculated the response matrices of a homogeneous slab of a thickness $5 \mathrm{~cm}$ and next determined the reflection matrix of a slab of the same composition with a thickness $25 \mathrm{~cm}$ from the above matrices by means of the combination rule. Then the result was compared with that by the direct calculation for a slab of a thickness $25 \mathrm{~cm}$.

Table 1 shows that reflection matrices by both methods coincide with each other.

Table 1 Reflection matrix of a slab

\begin{tabular}{c|c|c}
\hline & Direct calculation & $\begin{array}{l}\text { Indirect calculation } \\
\text { by means of } \\
\text { combination rule }\end{array}$ \\
\hline$R_{\mathbf{1 , 1}}$ & 0.89023881 & 0.89023880 \\
\hline$R_{\mathbf{1}, \mathbf{2}}$ & 0.59211442 & 0.59211441 \\
\hline$R_{\mathbf{2 , 1}}$ & 0.08742613 & 0.08742614 \\
\hline$R_{\mathbf{2}, 2}$ & $\mathbf{0 . 6 3 1 9 3 8 7 8}$ & 0.63193879 \\
\hline
\end{tabular}

Composition: TTR core, Thickness: $25 \mathrm{~cm}$

\section{Check calculation 2}

(Bare reactor calculation)

The critical thickness of a bare homogeneous slab reactor was first determined by the bare reactor theory with the boundary condition that flux should vanish at an extrapolated boundary. This reactor was next divided into 4 regions on purpose and the criticality calculation was performed by the response matrix method. The reflection matrix of the outer region was calculated under the boundary condition at the outer surface that flux should vanish at the extrapolated boundary so as to be consistent with the bare reactor theory. Table 2 shows that

Table 2 Comparison with bare reactor theory

\begin{tabular}{l|c|c}
\hline & $\begin{array}{l}\text { Bare reactor } \\
\text { theory }\end{array}$ & $\begin{array}{l}\text { Response matrix } \\
\text { method }\end{array}$ \\
\hline $\begin{array}{l}\text { Multiplication } \\
\text { factor }\end{array}$ & 1.0000000 & 1.0000000 \\
\hline $\begin{array}{l}\text { Thermal to } \\
\text { fast flux }\end{array}$ & 0.23027063 & 0.23027057 \\
\hline Composition: TTR core, Thickness: $47.151114 \mathrm{~cm}$ \\
\hline
\end{tabular}

* USSC-90 is the medium scale computer made by Remington Rand Co. The execution speed of this computer with the interpreter is about same to that of IBM 650 with the floating device. 
the response matrix method gives us the exact solution.

3. Check calculation 3 (Comparison with the finite difference method)

The criticality calculation of a bisymmetrical slab reactor consisting of 3 regions, a core, a graphite reflector and a water reflector was performed both by the response matrix method and by the finite difference method. The core thickness was adjusted so that the reactor may be just critical. The number of mesh points in the finite difference method is selected in three ways 25,50 and 95 , since the accuracy of the finite difference method

Table 3 Comparison with finite difference method

\begin{tabular}{|c|c|c|c|c|c|}
\hline & & \multicolumn{3}{|c|}{ Finite difference method } & $\begin{array}{l}\text { Response } \\
\text { matrix } \\
\text { method } \\
\end{array}$ \\
\hline & & \multicolumn{3}{|c|}{ USSC-90 } & USSC-90 \\
\hline \multicolumn{2}{|c|}{$\begin{array}{l}\text { Name of } \\
\text { code }\end{array}$} & \multicolumn{3}{|c|}{$\mathrm{SIC}^{\dagger}$} & \begin{tabular}{l|l} 
MER- \\
MAID-IA
\end{tabular} \\
\hline \multicolumn{2}{|c|}{$\begin{array}{l}\text { Output of } \\
\text { code }\end{array}$} & \multicolumn{3}{|c|}{$\begin{array}{l}\text { Flux distribution, power } \\
\text { distribution, multiplication } \\
\text { factor }\end{array}$} & $\begin{array}{l}\text { Current at } \\
\text { interface, } \\
\text { mean flux, } \\
\text { multiplica- } \\
\text { tion factor }\end{array}$ \\
\hline \multicolumn{2}{|c|}{$\begin{array}{l}\text { Number of } \\
\text { mesh points }\end{array}$} & 25 & 50 & 95 & \\
\hline $\begin{array}{c}\text { Execu- } \\
\text { tion } \\
\text { time }\end{array}$ & \begin{tabular}{rl|}
$t=1$ \\
$t=$ \\
$t=4$
\end{tabular} & $\begin{array}{r}15 \mathrm{~min} \\
6 \mathrm{~min}\end{array}$ & $\begin{array}{l}25 \mathrm{~min} \\
10 \mathrm{~min}\end{array}$ & $\begin{array}{l}50 \mathrm{~min} \\
20 \mathrm{~min}\end{array}$ & $15 \mathrm{sec}$ \\
\hline 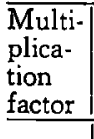 & & & $\begin{array}{l}1.000531 \\
1.000548\end{array}$ & $\begin{array}{l}1.000149 \\
1.000566\end{array}$ & 1.000000 \\
\hline \multirow{6}{*}{$\begin{array}{l}\text { Flux } \\
\text { at } \\
\text { inter- } \\
\text { face }\end{array}$} & $\begin{array}{r}\mathrm{ttf} \\
\phi_{1}(0)\end{array}$ & 1.00000 & 1.00000 & 1.00000 & 1.000000 \\
\hline & $\phi_{2}(0)$ & 0.23034 & 0.23033 & 0.23032 & 0.230315 \\
\hline & $\phi_{1}(1)$ & 0.54287 & 0.54179 & 0.54125 & 0.541026 \\
\hline & $\phi_{2}(1)$ & 0.20002 & 0.19844 & 0.19753 & 0.197161 \\
\hline & $\phi_{1}(2)$ & 0.16590 & 0.17060 & 0.17177 & 0.172158 \\
\hline & $\phi_{2}(2)$ & 0.23431 & 0.22635 & 0.22327 & 0.222110 \\
\hline \multirow{2}{*}{$\begin{array}{l}\text { Mean } \\
\text { flux } \\
\text { in core }\end{array}$} & $\overline{\phi_{1}}$ & & & 0.84862 & 0.848535 \\
\hline & $\bar{\phi}_{2}$ & 0.20513 & 0.20461 & 0.20444 & 0.204361 \\
\hline
\end{tabular}

Geometry: bisymmetrical slab reactor

Composition: Region 1 TTR core,

Region 2 graphite reflector,

Region 3 water reflector

- Programmed by Mr. Tokizawa of NAIG Co

it $t$ represents the number of source iterations.

tit $\phi_{1}(n)$ represents fast flux at the interface between region $n$ and region $n+1$ and $\phi_{2}$ represents thermal flux. depends upon the number of mesh points. The result is shown in Table 3 . The multiplication factor and neutron flux by the finite difference method converge uniformly to those by the response matrix method with the increased number of mesh points. It may be concluded from this convergency that the respanse matrix method gives us the exact solution. The execution time required for the calculation by the present method is significantly smaller than that by the finite difference method. The difference in execution time between both methods becomes much larger in the criticality calculations of reactors consisting of various combinations of spatial regions, since the calculation of the response matrix of a region is required only once.

\section{Criticality Calculation by Electronic Analog Computer}

We shall next solve Eq. (1) by use of an electronic analog computer. An analog element shown in Fig. 4 simulates the response characteristics of a one-dimensional region with two group approximation. A simulator for a region requires for 16 potentiometers and 4 adders.

Simulators for the right sub-assembly and the left one are easily obtainable by connecting those simulators for regions. Criticality calculation can be performed by connecting the simulator for the right sub-assembly with that for the left one so that equations

$$
j_{n}^{+} / j_{1}^{+}=j_{n}^{+} / j_{1}^{+} \quad n=2,3, \cdots \cdots, G
$$

may hold, where $j_{n}^{+}$represents the incident current of the group $n$ to the right subassembly and $j_{n}^{+}$, the reflected current from left one. The multiplication factor is expressed as

$$
\lambda=\bar{j}_{i}^{+} / j_{1}^{+} .
$$

For a bisymmetrical reactor the simulator for the right sub-assembly is sufficient for the calculation.

A block diagram for a criticality calculation of a bisymmetrical reactor with two-group approximation is shown in Fig. 5. The integrator $J_{1}$ in the figure has the role to prevent 


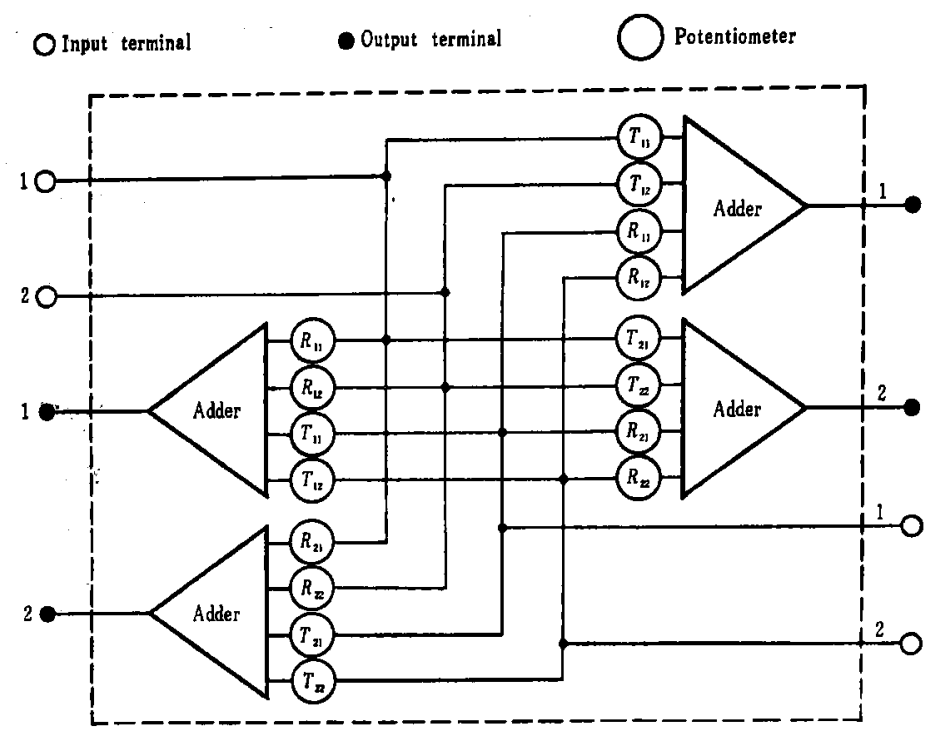

Fig. 4 Simulator for a one-dimensional region

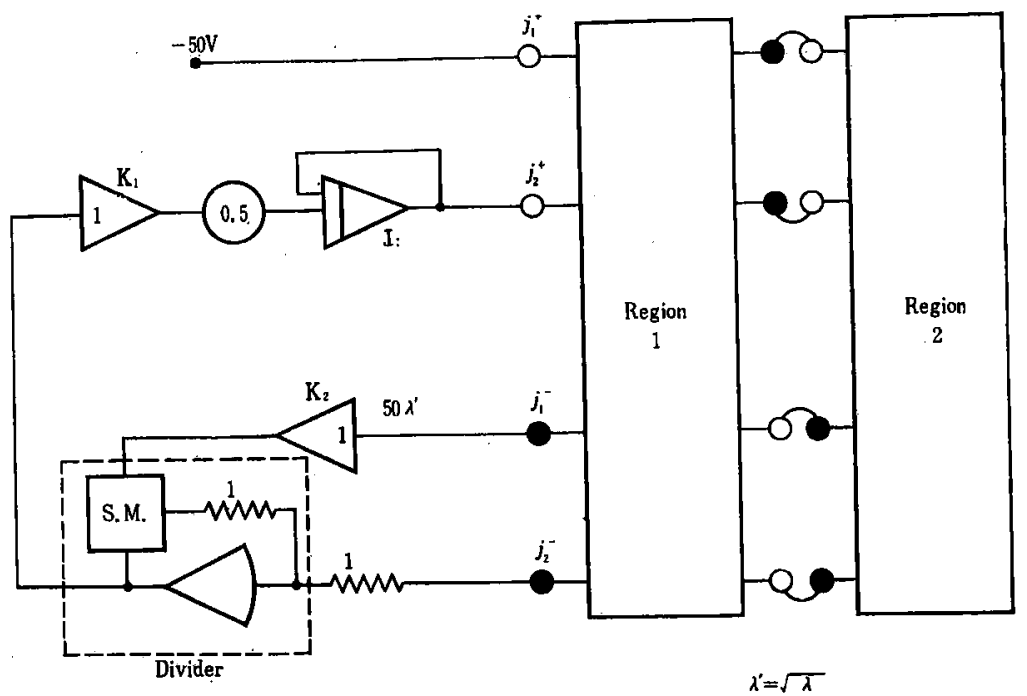

Fig. 5 Block diagram for criticality calculation with two-group

the instability in the solution of an eigenvalue problem, which some times occurs due to the loop of the electronic elements. Since every element of the reflection matrix is always positive, it may be proved that integrator makes the analog solution stable.

In order to confirm the accuracy of the analog calculation, a comparison with the calculation by a digital computer was made. Table 4 shows that the analog calculation gives us the multiplication factor of two- region reactors within $0.1 \%$ error. The error increases up to $0.5 \%$ for four-region reactors. Compared with other criticality calculations by usage of an electronic analog computer ${ }^{(5)}$, the present calculation can achieve comparatively high accuracy with rather simple procedure. Further, since the coefficients to be adjusted by potentiometers have the clear physical meaning, the present method is useful to evaluate the effect of the reflector or the control plate upon the criticality. 
Table 4 Multiplication factor of reflected reactor

\begin{tabular}{l|r|r}
\hline $\begin{array}{l}\text { Reflector } \\
\text { composition }\end{array}$ & $\begin{array}{l}\text { By analog } \\
\text { computer }\end{array}$ & $\begin{array}{l}\text { By digital } \\
\text { computer }\end{array}$ \\
\hline $\mathrm{H}_{2} \mathrm{O}$ & 0.992 & 0.9924 \\
$\mathrm{D}_{2} \mathrm{O}$ & 1.019 & 1.0192 \\
$\mathrm{Be}$ & 1.060 & 1.0596 \\
$\mathrm{C}$ & 1.017 & 1.0172 \\
$\mathrm{Al}$ & 0.960 & 0.9597 \\
$\mathrm{Fe}$ & 1.055 & 1.0547 \\
$\mathrm{Zr}$ & 1.008 & 1.0090 \\
$\mathrm{BeO}$ & 1.060 & 1.0598 \\
\hline
\end{tabular}

Core composition: TTR core

Thickness: $\quad 36.65 \mathrm{~cm}$

Reflector thickness: $10 \mathrm{~cm}$

† TOSAC 』 made by Toshiba Co.

\section{Conclusion}

The response matrix mothod has successfully been applied to the criticality calculation of one-dimensional reactor. It has been shown that the method gives us the rigorous solution of the group-diffusion equation and that the execution time required for the calculation is significantly smaller than that of codes based on the finite difference method.

Compared with other analytical methods ${ }^{(6)(7)}$ the present method has a feature that the reactor consisting of many spatial regions can be treated without difficulty. The execution time required is simply proportional to the number of regions by the present method, whereas it is proportional at least to the square of the number of regions by a direct analytical method.

Compared with the finite difference method, the present method has the following features.

(1) The execution time required is signifcantly smaller for calculations with few energy groups.

(2) The multiplication factor can be first determined and flux is next obtainable if required. Hence the present method is convenient to the calculation for the criticality search.

(3) Regions to which diffusion approximation can not be applicable can be treated more straightforwardly by the present method.

(4) Neutron current at every interface between regions as well as neutron flux is rigorously obtainable.

It is possible to program a code by the present method such that it is as flexible as that by the finite difference method except that the number of energy groups should be fixed in our method.

One of the authors, T. Miyamoto, has been programming a more flexible code than MERMAID.IA for IBM 7090 .

It is also possible to apply the present method to the criticality calculation of two or three-dimensional reactcr.

We wish to express our deep gratitude to Mr. R. Wakabayashi for his constant support of this work and to staffs of Reactor Theory Section of NAIG Nuclear Research Lab. for their many helpful discussions.

(Received February 4, 1963)

$$
\text { - REFERENCE- }
$$

(1) A. SHimizu: 'Response Matrix Method', in this issue.

(2) R. EHRLICH, H. HuRwitz: Nucleonics, 2, No. 12, 23 (1954).

(3) E. L. Wachspress, P. M. Stone, C. E. Lee: A/Conf., 15/P/633, (1958).

(4) A. M. Weingerg, E. P. Wigner: "The Physical Theory of Neutron Chain Reactors", Chap. XII, (1958), The Univ. of Chicago Press.

(5) J. G. Bayly, R. M. Pearce: Nucl. Sci. Engng., 2, 352 (1957).

(6) S. Glasstone, M. C. Edlunds: "The Elements of Reactor Theory", (1952), Van Norstrand, New York.

(7) A. Foderaro, H. L. Garabedian: Nucl. Sci. Engng., 8, 44 (1960). 